\title{
El rol de la paradiplomacia en las entidades binacionales: análisis del accionar de las provincias argentinas y regiones chilenas en los casos de EBITAN y EBIFETRA*
}

\author{
The role of paradiplomacy in binational entities: analysis of the \\ Argentinean provinces and Chilean regions actions in the cases of \\ EBITAN and EBIFETRA
}

\author{
Mariano Álvarez ${ }^{* *}$ \\ Instituto de Estudios Internacionales, Universidad de Chile \\ Recibido: 1 de abril de 2017. Aprobado: 17 de mayo de 2017
}

\begin{abstract}
Resumen
Desde fines de los ochenta, Argentina y Chile han comenzado un proceso de integración que se vio favorecido con el regreso a la democracia. Dentro del mismo, el componente subestatal se ha ido incorporando, especialmente a partir del Tratado de Maipú de 2009, entregando mayores oportunidades de participación a los gobiernos no centrales. No obstante, es necesario explorar si, más allá del reconocimiento por parte de los Estados, las provincias argentinas y regiones chilenas han sabido aprovechar los canales de participación que se les presentan. A tal fin se analizan comparativamente las entidades binacionales creadas para los proyectos Túnel Internacional de Agua Negra (EBITAN) y Ferrocarril Trasandino Central (EBIFETRA).
\end{abstract}

Palabras clave: paradiplomacia, entidades binacionales, integración.

El presente artículo resulta de la tesis doctoral "Paradiplomacia en las relaciones chileno-argentinas: La integración desde Coquimbo y San Juan”, defendida en la Universidad de Leiden el año 2016 y financiada con Becas Chile.

** Doctor en Humanidades, Universidad de Leiden; Magíster en Estudios Internacionales, Universidad de Chile; Licenciado en Relaciones Internacionales, Pontificia Universidad Católica Argentina; Licenciado en Ciencias Políticas, Pontificia Universidad Católica Argentina. Profesor Visitante del Instituto de Estudios Internacionales de la Universidad de Chile. Correo electrónico: marianoalv@gmail.com 


\begin{abstract}
Since the end of the eighties, Argentina and Chile have begun a process of integration, favored by the return to democracy. Since then, the sub-state component has been incorporated in the process, especially through the Maipú Treaty of 2009, offering the non-central governments greater opportunities of participation. Nonetheless, it is necessary to explore whether the Argentinean provinces and the Chilean regions have exploited the channels of participation offered to them by the states. To this end, this paper analyzed the binational entities created for the projects Túnel Internacional de Agua Negra (EBITAN) and Ferrocarril Trasandino Central (EBIFETRA), comparatively.
\end{abstract}

Keywords: paradiplomacy, binational entities, integration.

\title{
Introducción
}

El fenómeno paradiplomático es cada vez más recurrente en América Latina (véase Maira, 2010), donde los gobiernos no centrales se han involucrado tanto de forma directa como indirecta en asuntos exteriores. Si bien dichas actividades se han consolidado recientemente, su estudio es aún más nuevo, ya que para mediados de los dos mil, con la excepción de México y de centros académicos puntuales como la Universidad Arturo Prat, en América Latina no se le ha dado mayor relevancia (Ferrero, 2005). Los primeros trabajos sobre paradiplomacia ${ }^{1}$ se generaron en Norteamérica durante la segunda mitad de la década de los ochenta ${ }^{2}$, expandiéndose primero por la academia europea durante los noventa ${ }^{3}$ y recién con el nuevo milenio comenzaron a arribar de manera más decidida

1 En el presente artículo, el término paradiplomacia seguirá la definición propuesta en Álvarez (2016), según la cual consiste en "el involucramiento, directo o indirecto, en actividades permanentes o ad hoc, de los gobiernos no centrales en el núcleo blando de los asuntos exteriores" (p. 49).

2 El punto de inflexión en los estudios sobre paradiplomacia suele colocarse en la publicación del número de otoño de 1984 de la revista Publius, donde se encontraba contenido el trabajo de Ivo Duchacek (1984). No obstante, este se insertaba dentro de una corriente de pensadores donde se encontraban a su vez, Kincaid (1990), Michelmann y Soldatos (1990) y Hocking (1986) entre los principales exponentes.

3 La academia europea incorporó paulatinamente el tópico, hasta convocar en 1997 a un seminario de expertos que resultó en la publicación de un número especial de la revista Regional \& Federal Studies, donde se incluyeron los trabajos de Aguirre (1999), Cornago (1999) y Keating (1999) entre los principales. Asimismo, junto con los trabajos de cinco autores más, se publicó el libro de Aldecoa y Keating (2000), impreso originalmente en inglés en 1999. 
a Sudamérica ${ }^{4}$ (Kuznetsov, 2014). Con ello se abrió un gran abanico de posibilidades para expandir los estudios internacionales, incorporando al actor subestatal.

Dentro del nuevo campo de estudio, el análisis de la actividad paradiplomática puede ser abordado desde diversas ópticas: estudiando sus causas ${ }^{5}$; sus motivaciones y objetivos ${ }^{6}$; la forma en que se lleva a cabo; o el canal que utiliza, por mencionar las más frecuentes. En el último de los casos, el estudio se divide fundamentalmente en dos grandes vertientes, a saber: analizar los canales paradiplomáticos informales o aquellos ya institucionalizados. En el presente artículo se espera arrojar luz sobre las diferencias que presentan los gobiernos no centrales en su aprovechamiento de los canales paradiplomáticos formales establecidos entre Argentina y Chile. Para hacerlo, se han seleccionado los casos de las entidades binacionales Túnel Internacional Paso de Agua Negra (EBITAN) y Túnel de Baja Altura-Ferrocarril Trasandino Central (EBIFETRA).

El primer apartado del presente artículo presentará un breve marco teórico y detallará la metodología seguida para el análisis. El segundo apartado se abocará al comportamiento seguido por Argentina y Chile al momento de reconocer el fenómeno paradiplomático. La tercera sección recogerá el proceso por el cual los dos países han institucionalizado las acciones paradiplomáticas coordinadas. El cuarto apartado analizará cómo, ante una misma oportunidad de involucramiento paradiplomático, no se ha producido un aprovechamiento similar entre los distintos gobiernos no centrales. Como hipótesis se propone que el desarrollo del accionar paradiplomático a través de los canales formales no depende exclusivamente de estos, sino del aprovechamiento que los gobiernos no centrales hagan de los mismos. Finalmente, el artículo concluye con un corolario en que se recogen las principales ideas esgrimidas en el texto.

4 En América Latina no se produjo un giro marcado por un acontecimiento o publicación, como sí ocurriese en Norteamérica o Europa. Ejemplo de ello es que, en la región, Bernal Meza fue un precursor de los estudios paradiplomáticos con su trabajo de 1990; sin embargo, la corriente de pensamiento no se expandió rápidamente y la siguiente publicación se produjo recién cuatro años más tarde con Colacrai y Zubelzú (1994), quienes no mencionan el artículo de Bernal Meza. No obstante, el estudio de la paradiplomacia se continuó desarrollando y para 2002 el Consejo Argentino para las Relaciones Internacionales (CARI) creó el Comité sobre las Provincias en el Plano Internacional, desde donde se publicaron los informes de Zubelzú e Iglesias (2005a, 2005b) y el libro de Iglesias, Zubelzú e Iglesias (2008). Asimismo, en 2009 se llevó a cabo un seminario internacional referido a la paradiplomacia en América Latina, del cual resultó el libro editado por Maira (2010).

$5 \quad$ Véanse los trabajos de Bernal Meza (1990) y Tussie (2004), entre otros.

6 Véanse los trabajos de Kincaid (2003), Keating (1999, 2000, 2003) y Michelmann (1990, 2009), entre otros. 


\section{Enfoque teórico y descripción de la metodología}

Existen al menos cuatro grandes tipos de acciones paradiplomáticas (Zubelzú e Iglesias, 2005b). Un primer tipo son las acciones propias, que se dan de forma directa en el extranjero. El segundo grupo de acciones es el lobby interno, donde los gobiernos no centrales buscan inducir a su Estado para que actúe internacionalmente de una manera determinada. El tercer tipo de acciones son las concertadas, se dan dentro de un marco y una normativa dada por el gobierno central, pero sin que este intervenga. Finalmente, el cuarto tipo de acción es la coordinada con el gobierno central, donde a través de canales que este propone, los gobiernos no centrales pueden trabajar en conjunto con el central en temas internacionales.

Nos proponemos aquí analizar el último tipo de acción a través del estudio del accionar paradiplomático en las entidades binacionales desarrolladas por Argentina y Chile. A fin de realizar dicha labor, el presente artículo propone un acercamiento desde el neoinstitucionalismo de acción racional, donde "el comportamiento de cada actor es una respuesta óptima al comportamiento de los otros jugadores y a la estructura institucional existente" (Tsebelis, 1990, p. 92). Ello implicará observar cómo la agencia ha influido en el agente, al tiempo que este modificó la primera, ya que si bien las instituciones indican "qué conductas o situaciones son requeridas, prohibidas o permitidas" (Zurbriggen, 2006, p. 69), no determinan los intereses ni los esquemas de preferencias que los agentes deben seguir.

A través de dicho marco teórico, se observará en primer lugar cómo los países han establecido una institucionalidad dada - las entidades binacionales - y qué rol le asignaron estas a los gobiernos no centrales ${ }^{7}$. En segundo lugar, se explorará el efecto que los agentes - provincias argentinas y regiones chilenas - tuvieron sobre las instituciones. Ello permitirá comparar el desarrollo de dos agencias -EBITAN y EBIFETRA-, que fueron establecidas con una institucionalidad idéntica, pero cuyos componentes paradiplomáticos, de acuerdo con la hipótesis planteada, las hicieron desarrollarse de forma diferenciada.

El análisis será abordado a través de dos pilares metodológicos. El primero es un estudio documental del devenir de los instrumentos jurídicos establecidos por Argentina y Chile;

El presente artículo utilizará el concepto de gobierno no central para hacer referencia a las provincias argentinas y regiones chilenas. 
en tanto que el segundo es un acercamiento al rol de los agentes por medio de ocho entrevistas a informantes clave.

Respecto al componente jurídico, a fin de describir el desarrollo de las entidades binacionales en tanto instituciones, el artículo presenta un análisis de los diversos instrumentos internacionales que Argentina y Chile acordaron en su proceso de integración ${ }^{8}$ y que sentaron las bases para la construcción del andamiaje jurídico sobre el cual se propuso la creación de las entidades binacionales. Asimismo, se explorarán los instrumentos constitutivos de EBITAN y EBIFETRA, los protocolos que los modificaron y los reglamentos que se establecieron para su funcionamiento, a fin de presentar el desarrollo de la agencia y cómo fue cambiando en esta el rol del agente.

En lo referido al accionar de las provincias y regiones, debido a la escasez de información documentada al respecto, en especial sobre las discusiones al interior de las entidades binacionales y las motivaciones de los gobiernos no centrales en estas, se recurrió a entrevistar informantes clave. Se realizaron ocho entrevistas semiestructuradas: Alejandro Corvalán, ex-secretario Regional Ministerial de Economía en Valparaíso; Ezequiel Páez, secretario asesor en la Embajada de Argentina en Chile; Gabriela Stortoni, miembro de la EBITAN por parte del Ministerio de Planificación Federal, Inversión Pública y Servicios; Gastón Díaz, encargado de la Oficina de Relaciones Internacionales de Coquimbo; Iván Páez, jefe de la Unidad de Asuntos Internacionales de la Dirección Nacional de Fronteras y Límites del Estado (DIFROL); Luis Maira, ex-embajador de Chile en Argentina; Rigoberto García, coordinador internacional del Ministerio de Obras Públicas de Chile; y Santiago Melo, agregado de la DIFROL en la Embajada de Chile en Argentina. Sobre la base de su información se procedió al análisis del agente y cómo este se había desempeñado dentro de las entidades binacionales, aprovechando o no las oportunidades paradiplomáticas que se le ofrecieron a las provincias y regiones.

\section{Reconocimiento del fenómeno paradiplomático en Argentina y Chile}

Los dos países del extremo sur del continente tuvieron, al interior de sus estructuras gubernamentales, desarrollos institucionales distintos respecto a la paradiplomacia. Ello se explica en parte debido a las diferencias en sus configuraciones políticas (tanto

8 En el presente artículo se entenderá por integración el incremento en la interdependencia de los dos países, sin distinción de sector ni necesidad de supranacionalidad. 
la forma de Estado como el centralismo) (Álvarez, 2016). Sin embargo, ambos acordaron el desarrollo de una institucionalidad bilateral para que sus gobiernos no centrales interactuasen directamente respecto a sus problemas.

La formalización del accionar paradiplomático entre ambos países tuvo sus cimientos en la firma del Tratado de Paz y Amistad de 1984, lo cual se consolidó posteriormente con el fin de los gobiernos militares. Gracias a ello, la actividad paradiplomática aumentó significativamente entre Argentina y Chile durante la década de los dos mil (véase Silva Soura y Morán León, 2010). De acuerdo con Santiago Melo9, las actividades relativas a la integración estuvieron vedadas durante finales de los setenta y principios de los ochenta, momento en que no se concebían, por ejemplo, obras de infraestructura de interconexión. La razón de ello era la imperante lógica de desconfianza que se había instalado entre los países, lo cual también obstaculizaba cualquier desarrollo de iniciativas conjuntas a nivel subestatal. El cambio se comenzó a producir gracias a la distensión militar propiciada por el tratado de 1984, a lo cual se sumó la llegada de Patricio Aylwin (1990-1994) como primer presidente democrático en Chile luego del gobierno militar. Ello limaba las asperezas que habían quedado luego del tratado, ya que el presidente argentino Raúl Alfonsín (1983-1989) mantuvo una relación tensa con Augusto Pinochet (1974-1990) (Church, 2011).

A la normalización de las relaciones se agregó posteriormente el impulso a la integración de Chile con América Latina, promovida durante la administración de Ricardo Lagos (2000-2006). Originalmente, las regiones chilenas se habían internacionalizado en búsqueda de cooperación y hermanamiento, pero bajo el marco de la integración se le empezó a dar mayor importancia a temas de infraestructura, como los corredores bioceánicos, lo que inclusive llevó a la creación de la Comisión Asesora Presidencial para la Cooperación Internacional en Regiones (Schnake, 2011). De dicha forma, la distensión militar lograda con el tratado de 1984, sumada a las nuevas relaciones en democracia, a la reforma constitucional de 1994 en Argentina ${ }^{10}$ y al enfoque de Lagos en Chile, hicieron que durante la década del dos mil las provincias y regiones tuviesen un mayor involucramiento en los asuntos exteriores.

Con el cambio de milenio, las actividades entre las provincias y regiones se intensificaron. Ejemplo de ello fueron las misiones binacionales organizadas desde la Región de

$9 \quad$ Entrevistado por el autor en Buenos Aires el 6 de noviembre de 2014.

10 Los alcances de la reforma constitucional y del nuevo artículo 124, si bien de gran relevancia para el desarrollo paradiplomático de las provincias, escapan a los fines del presente artículo. El lector puede consultar a Gasol Varela (2012), Natalizio (2007) y María Hernández (2003), entre otros. 
Valparaíso. Recuerda Alejandro Corvalán ${ }^{11}$ que el proceso de integración con la provincia de Mendoza partió en 1999, pero se consolidó los años siguientes. Para ello se idearon y ejecutaron los primeros foros de desarrollo productivo binacionales, en los que además de la Región de Valparaíso y la provincia de Mendoza también se invitó a la provincia de San Luis. A partir de dichos foros, se desarrollaron en 2001 y 2004 las primeras misiones conjuntas de gobiernos no centrales argentinos y chilenos en terceros países, lo que constituyó un hecho inédito.

No obstante, si bien en Chile se produjo un cierto reconocimiento de espacios para el desarrollo paradiplomático, no siempre se ha sabido o querido incorporar al componente subestatal en la política exterior oficial (Schnake, 2011). Como resultado de ello, no solo se ha generado cierta resistencia al desarrollo externo de las regiones; también se han producido desarrollos heterogéneos entre ellas, en especial debido los distintos ritmos de aprendizaje y de interés que las mismas presentan.

Por su parte, el involucramiento más decidido de las provincias argentinas en asuntos exteriores tampoco se desarrolló sin inconvenientes. De acuerdo con el segundo informe del proyecto "Provincias y Relaciones Internacionales" (Zubelzú e Iglesias, 2005b), para inicios de los dos mil, los mayores problemas se relacionaban con cuestiones procedimentales u operativas, destacándose en especial la falta o debilidad de la coordinación entre los distintos niveles de gobierno. Asimismo, el escaso conocimiento que las unidades subestatales tenían de las oportunidades, limitaciones y parámetros de la paradiplomacia obstaculizaba su desarrollo internacional.

Aun así, tanto las regiones como las provincias se fueron involucrando en los asuntos exteriores de sus países, dificultando los intentos por imponer una política exterior pensada únicamente desde el centro. Sin embargo, el rol desempeñado por las entidades subestatales a uno y otro lado de la cordillera ha sido distinto, siendo las argentinas normalmente más activas. Una hipótesis respecto a las diferencias entre Argentina y Chile ha sido que el surgimiento de la paradiplomacia en el segundo se favoreció principalmente por políticas de regionalización impulsadas desde el centro, en tanto que en Argentina fueron las provincias las que reclamaron para sí mayor participación. Sin embargo, si bien es cierto que la actividad de las regiones se da más por oferta desde el gobierno central que por demanda desde las regiones (Cornago, 2010), los gobiernos no centrales también han presentado inquietudes e intereses específicos en asuntos exteriores. Las motivaciones propias para impulsar la paradiplomacia claramente han aumentado y,

Entrevistado por el autor en Valparaíso el 4 de noviembre de 2014. 
como señala Gastón Díaz ${ }^{12}$, el nivel central en gran medida ha debido institucionalizar las relaciones internacionales para facilitar la participación, que ya se daba desde las regiones y comunas.

Con ello se quiere ejemplificar que, aun a pesar de las diferencias en las configuraciones políticas de ambos países y de la injerencia que esto tiene sobre las posibilidades paradiplomáticas (véase Álvarez, 2016), el involucramiento de las provincias argentinas y regiones chilenas en asuntos exteriores ha ido en aumento a partir de la década del noventa, y en especial a partir del cambio de milenio. Ello, como se verá, impulsó también a los países a cristalizar las iniciativas en instituciones bilaterales; el siguiente apartado analiza los pasos que Argentina y Chile siguieron para crear un espacio de acciones coordinadas a nivel bilateral.

\section{Desarrollo del marco bilateral para acciones coordinadas}

Con el Tratado de Paz y Amistad de 1984 se plantó el germen de la institucionalidad paradiplomática entre Argentina y Chile, pero fue recién con el retorno a la democracia que los países consolidaron sus vínculos y se comenzó a crear un marco institucional bilateral para la integración. Es dentro de dicho cuerpo de tratados que se gestó el espacio para que las provincias y regiones se incorporasen al proceso.

Argentina y Chile habían comenzado a litigar respecto al límite que los separaba durante la segunda mitad del siglo XIX; el grueso del diferendo había sido saldado en 1902 y durante las décadas que siguieron se llevó a cabo el lento proceso de consolidación de los hitos. Si bien dentro de dicho proceso existieron diferendos puntuales (como Alto Palena y Laguna del Desierto), fue el conflicto por el canal Beagle el que colocó a los países en pie de guerra durante la década de los setenta. Por ello, el objetivo central del Tratado de Paz y Amistad de 1984 era muy simple: solucionar el conflicto limítrofe originado por el canal Beagle entre los dos países. Sin embargo, la oportunidad fue también aprovechada para sentar las bases del proceso de acercamiento e integración que partiría en los años siguientes.

Se trató de un instrumento complejo y de gran relevancia para ambos pueblos. A los fines del presente trabajo lo que interesa en especial es su artículo 12, que es el primero de la sección de Cooperación Económica e Integración Física. Allí se crea la Comisión

12 Entrevista escrita, recibida por el autor el 19 de enero de 2015. 
Binacional, que será la encargada de promover y desarrollar iniciativas de enlace terrestre, puertos y zonas francas, entre otros; ella será la que, posteriormente, dará forma al proceso de institucionalización de la paradiplomacia a través de los comités de frontera, que en 2006 pasarán a llamarse comités de integración.

Si bien podemos considerar al Tratado de Paz y Amistad de 1984 como la piedra angular de la nueva relación entre Argentina y Chile, el que realmente produjo el despegue institucional de las relaciones paradiplomáticas fue el Tratado de Maipú de Integración y Cooperación, firmado en 2009 para conmemorar los veinticinco años del Tratado de Paz y Amistad. De acuerdo con Artaza Rouxel (2010), en la declaración conjunta de las presidentas Cristina Fernández (2007-2015) y Michelle Bachelet (2006-2010), estas "se felicitaron por los importantes logros obtenidos en materia de integración y cooperación entre las regiones chilenas y las provincias argentinas, la conformación de siete Comités de Integración y los más de treinta mecanismos e instancias bilaterales" (p. 360). Si bien Luis Maira ${ }^{13}$ recuerda que junto con su par Ginés González García debieron intervenir fuertemente para que sus ministerios de Relaciones Exteriores aceptasen incorporar el componente paradiplomático en el acuerdo, el apoyo de las mandatarias fue lo que motivó que finalmente se incluyese un capítulo al respecto. Inclusive, el artículo primero del Tratado de Maipú, el cual contiene los objetos del mismo, terminó dándole una gran importancia al componente subestatal, ya que en tres de los objetivos se reconoce la relevancia de los gobiernos no centrales en la relación bilateral.

El tratado no solo estableció los canales institucionales para la paradiplomacia: en el análisis de Ezequiel Páez ${ }^{14}$, el carácter de documento bilateral firmado al más alto nivel otorgó al Tratado de Maipú el rol de marco internacional legitimador. En otras palabras, las instituciones que allí se incluyeron, ya fuese en su articulado o en sus protocolos adicionales, fueron el resultado de decisiones validadas desde su concepción por los gobiernos centrales. De dicha forma, se creó un paraguas político bajo el cual se dejó de considerar a las provincias y regiones como entidades que se inmiscuyesen en asuntos exteriores, pasando a ser consideradas como actores legítimos del proceso de integración.

Dentro de dicho marco institucional se legitimó especialmente a los comités de integración como "foros de encuentro y colaboración entre los sectores público y privado de las provincias argentinas y regiones chilenas para promover la integración en el ámbito subnacional, con el apoyo de los organismos nacionales, provinciales, regionales

3 Entrevistado por el autor en Santiago de Chile el 13 de enero de 2015.

14 Entrevistado por el autor en Santiago de Chile el 26 de noviembre de 2014. 
y municipales" (Tratado de Maipú de Integración y Cooperación, 2009, p.7). Asimismo, el artículo 18 facultó a las provincias y regiones a, actuando en forma conjunta en el seno de los comités de integración, proponer de manera directa iniciativas específicas a la Comisión Binacional de Cooperación Económica e Integración Física, así como a la Comisión Binacional de Comercio, Inversiones y Relaciones Económicas.

Sin embargo, lo más relevante para el presente análisis es que el artículo 24 del Tratado de Maipú abrió la puerta a la creación de entidades binacionales. Gracias a ello, en conjunto con la firma del tratado se suscribieron los protocolos complementarios para la creación de la Entidad Binacional Túnel de Baja Altura-Ferrocarril Trasandino Central (EBIFETRA) y la Entidad Binacional Túnel Internacional Paso de Agua Negra (EBITAN).

De esta forma, a través del Tratado de Maipú y de sus protocolos complementarios se establecieron dos mecanismos de acción paradiplomática, uno de los cuales son las entidades binacionales que serán analizadas en el apartado siguiente. El primero de los mecanismos fue la consolidación de los comités de integración, que habían nacido sobre la base del Tratado de Paz y Amistad de 1984, pero que habían avanzado con reformas ad hoc y sin un instrumento jurídico sólido que los validase internacionalmente como foros de participación subestatal. El segundo mecanismo fueron las mencionadas entidades binacionales, creadas en los protocolos adicionales y que era algo novedoso para Chile, donde, de acuerdo con Rigoberto García ${ }^{15}$, el país no tenía experiencia.

Finalmente, resulta importante resaltar que esta incorporación de las provincias y regiones en el Tratado de Maipú, si bien fue impulsada por los embajadores y las mandatarias, no fue algo que el gobierno central decidiese unilateralmente. De acuerdo con Iván Páez ${ }^{16}$, se trató tanto de un ofrecimiento de los Estados como de una cristalización de situaciones que impulsaron los gobiernos no centrales. En dicha lógica es que se entiende que uno de los objetivos estratégicos del Tratado de Maipú fuese la consolidación y el fortalecimiento de las relaciones subestatales. Páez sostiene que lo que se hizo patente en el acuerdo fue la voluntad de aumentar las facultades de las provincias y regiones en temas internacionales, algo que además estaba en la línea de lo que los gobiernos no centrales venían impulsando. En otras palabras, si bien fue el gobierno central quien finalmente tomó la decisión de incorporar un capítulo sobre la participación de los gobiernos no centrales en el tratado bilateral, ello fue en respuesta a que las provincias y regiones manifestaron su interés por participar en el proceso de integración.

5 Entrevistado por el autor en Santiago de Chile el 12 de enero de 2015.

16 Entrevistado por el autor en Santiago de Chile el 28 de noviembre de 2014. 


\section{Las entidades binacionales Túnel de Agua Negra y Ferrocarril Trasandino Central}

Entre los objetivos del Tratado de Maipú se establecía la necesidad de mejorar y ampliar la interconexión física entre Argentina y Chile (artículo 1, inciso g). Al mismo tiempo, se acordaba que las partes podrían firmar protocolos complementarios que se refiriesen a materias específicas relacionadas con los objetivos del tratado (artículo 2). En línea con ello y tomando en consideración los posibles proyectos de infraestructura de transporte, el artículo 24 estableció que se podrían crear entidades binacionales de carácter público, las cuales serían responsables de "llevar adelante los procedimientos necesarios para la realización de los estudios técnicos, la elaboración de la documentación para la contratación de los trabajos, la construcción de las obras y su administración y operación posteriores" (p. 11). Argentina tenía experiencia en el desarrollo de este tipo de entidades con otros países limítrofes debido a la construcción de la represa de Yacyretá con Paraguay, pero se trataba de la primera vez que Chile se incorporaba a este tipo de instituciones. Como bien destaca Gabriela Stortoni ${ }^{17}$, fue un proceso complejo, ya que si bien existía voluntad política, para Chile las entidades binacionales eran una experiencia totalmente nueva, lo que hizo necesario un extenso trabajo de generación de confianza. Pero lo más relevante de las entidades binacionales para el presente análisis es que en ellas se comenzó a incorporar de forma oficial el trabajo coordinado entre los distintos niveles de gobierno en el ámbito internacional.

Gracias al paraguas legal que estableció el Tratado de Maipú y en especial a los mencionados artículos, se han acordado ya tres protocolos constitutivos de entidades binacionales, firmándose los de la Entidad Binacional Túnel Internacional Paso de Agua Negra (EBITAN) y de la Entidad Binacional Túnel de Baja Altura-Ferrocarril Trasandino Central (EBIFETRA) el mismo año del Tratado de Maipú. A través de ellos, los países depositaron una gran responsabilidad en las mencionadas entidades, debido a que según el artículo tercero de los protocolos de EBITAN y EBIFETRA, estas son las encargadas de:

(...) revisar los estudios técnicos necesarios para la factibilidad del proyecto, pudiendo realizar con ese objetivo los estudios y análisis adicionales y/o complementarios que estime convenientes en términos técnicos, económicos, financieros, ambientales y legales del Proyecto (para lo cual podrán) requerir de los organismos de ambos gobiernos toda la asistencia técnica y toda la información que considere necesaria para el cumplimiento de sus fines (Protocolo complementario al Tratado de Maipú

17 Entrevistada por el autor en Buenos Aires el 11 de noviembre de 2014. 
de Integración y Cooperación sobre la constitución de la Entidad Binacional para el proyecto “Túnel Internacional Paso de Agua Negra”, 2009, p.2).

A los fines del presente artículo, se destaca en especial que las entidades binacionales incorporaron desde su concepción la dimensión subestatal del lado argentino, al tiempo que abrieron las puertas para la ampliación de sus miembros. Es precisamente allí donde se han dado las diferencias entre el accionar de la Región de Coquimbo en EBITAN y la de Valparaíso en EBIFETRA. Tanto por oferta inicial de los Estados como por demanda específica de las provincias y regiones, la paradiplomacia se fue institucionalizando en acciones coordinadas, pero como se verá, la incorporación de los gobiernos no centrales siguió patrones diferenciados.

Respecto a la oferta inicial hecha desde los Estados, los artículos segundos de los protocolos complementarios para la EBIFETRA y EBITAN eran prácticamente idénticos. El de la primera establecía que esta estaría conformada:

(...) al menos, por seis miembros, tres de ellos designados por la República Argentina, a propuesta del Ministerio de Relaciones Exteriores, Comercio Internacional y Culto, del Ministerio de Planificación Federal, Inversión Pública y Servicios y del Gobierno de la Provincia de Mendoza; y tres designados por la República de Chile, a propuesta del Ministerio de Relaciones Exteriores y por el Ministerio de Obras Públicas. La entidad podrá incorporar nuevos miembros por consenso (Protocolo complementario al Tratado de Maipú de Integración y Cooperación sobre la constitución de la Entidad Binacional para el proyecto "Túnel de Baja Altura - Ferrocarril Trasandino Central", 2010, p.1).

Por otro lado, el protocolo de EBITAN solo se diferenciaba del anterior en establecer que los tres miembros designados por Chile serían uno a propuesta del Ministerio de Relaciones Exteriores y dos a propuesta del Ministerio de Obras Públicas.

Del artículo segundo de ambos protocolos se destaca que si bien se incluía el componente subestatal, esta aceptación de la paradiplomacia era asimétrica entre los países. Como queda en evidencia, en tanto Argentina daba cabida a las provincias de Mendoza y San Juan como miembros de las entidades binacionales, Chile dejaba a sus regiones relegadas de las nuevas instituciones.

Quienes se movilizaron, o pudieron haberlo hecho, para subsanar la omisión de las regiones en los protocolos, fueron los propios gobiernos no centrales. En la II Reunión 
de la Entidad Binacional Túnel Internacional Paso de Agua Negra, llevada a cabo en julio de 2010 y en la cual se estableció el reglamento para el funcionamiento de la EBITAN, el componente paradiplomático comenzó a ser reconocido. En primer lugar, es necesario resaltar que la presidencia de la EBITAN estuvo depositada en el ministro de Infraestructura y Tecnología de la provincia de San Juan, acompañado en la delegación argentina por la directora nacional de Países Limítrofes y el coordinador general de Vialidad Nacional. Con ello se depositaba la confianza en un representante directo de un gobierno no central, sobre el cual los ministerios nacionales no podían imponer su voluntad. En segundo lugar, la delegación de Chile estuvo conformada por el director nacional de Fronteras y Límites, el subdirector de Desarrollo de la Dirección Nacional de Vialidad y el secretario regional ministerial del Ministerio de Obras Públicas en Coquimbo. Si bien este último era de designación del gobierno central, estaba destinado al territorio de la región y fue el encargado de presentar una solicitud para incorporar, como representante permanente en la EBITAN, a un miembro del gobierno regional de Coquimbo. Esto significaba la modificación de una institución recién creada, para incorporar en ella un componente subestatal, representante de una región de un país unitario. No obstante, debido a lo involucrada que estaba la región, los ministerios no se opusieron a su incorporación, al tiempo que Argentina rápidamente accedió al pedido.

Cabe también resaltar que el artículo 11 del reglamento de la EBITAN estableció las sedes de la entidad en San Juan y La Serena, trasladando físicamente las decisiones a los territorios de los gobiernos no centrales. Además, el artículo 12 agregaba que la entidad se reuniría con la totalidad de sus miembros. Con ello se aseguraba que las voces de la provincia y de la región tenían que ser contempladas en el accionar de la entidad binacional.

A diferencia de lo ocurrido en la EBITAN, cuando en noviembre de 2010 se aprobó el reglamento de la EBIFETRA, este no incorporó a ningún representante del gobierno regional de Valparaíso. Si bien el artículo 18 del reglamento anunciaba que, de ser necesario, se podrían incorporar nuevos miembros, esto no se llevaba a la práctica. Asimismo, el artículo 17 fijó como sedes de la entidad a las ciudades de Buenos Aires y Santiago, lo cual ponía una gran distancia entre el gobierno provincial de Mendoza y la mesa de negociación. A ello se sumaba una profunda desprotección de los representantes mendocinos, debido a que el artículo 9 establecía que el órgano directivo de la entidad (el consejo) "podrá reunirse y/o adoptar decisiones válidas con la presencia mínima de dos consejeros de cada país" (p. 5). 
En definitiva, el reglamento de la EBIFETRA no solo dejaba de lado a la Región de Valparaíso, la cual no era considerada dentro de los miembros de la entidad, sino que además los ministerios nacionales podían reunirse en las capitales sin necesidad de esperar a los representantes de Mendoza. Con ello, la voz paradiplomática quedó seriamente comprometida en la entidad binacional.

Ambas entidades continuaron operando bajo la reglamentación establecida, hasta que en 2015 entraron en vigor los segundos protocolos complementarios al Tratado de Maipú. El relativo al proyecto Túnel Internacional Paso de Agua Negra incrementó la independencia de la EBITAN al otorgarle personalidad jurídica internacional y "capacidad jurídica, patrimonial, administrativa para adquirir derechos y contraer obligaciones y celebrar los actos y contratos que fueren necesarios para la consecución de su misión" (artículo 4, p. 3). No obstante, es necesario resaltar que no todos los cambios institucionales fueron en favor de una mayor participación subestatal. De acuerdo con el reglamento de 2010, las sedes de la EBITAN eran La Serena y San Juan (artículo 11), lo cual fue alterado en el II Protocolo Complementario, moviéndolas a la Ciudad Autónoma de Buenos Aires y Santiago (artículo 5) ${ }^{18}$.

El II Protocolo Complementario relativo al proyecto Túnel de Baja Altura-Ferrocarril Trasandino Central fue considerablemente distinto. En primer lugar, el lobby y avances logrados en EBITAN se habían reflejado en que su protocolo partiese "considerando que el Proyecto 'Túnel Internacional Paso de Agua Negra' resulta de interés estratégico para el proceso de integración binacional” (p. 1), algo que se encuentra ausente en el caso de EBIFETRA. No obstante ello, el protocolo también le entregó personalidad jurídica internacional a EBIFETRA, pero en lo relativo al componente subestatal, el protocolo no se apartó de lo señalado en su reglamento de 2010, manteniendo las sedes en Buenos Aires y Santiago, y sin mencionar a la Región de Valparaíso, si bien se recordaba la capacidad de la entidad para ampliar el número de sus miembros.

Lo que se destaca del desarrollo diferenciado de las dos entidades es que los involucrados desde los gobiernos centrales son los mismos actores en ambas, al tiempo que el objetivo es similar - la construcción de un túnel binacional-y la cantidad de actores subestatales es la misma - una provincia y una región-. Es por ello que resulta factible asignar una gran parte de la responsabilidad a Coquimbo y San Juan por haber logrado que la EBITAN tuviese una institucionalidad con mayor presencia subestatal que la que tuvo EBIFETRA.

18 Sin embargo, las sedes no siempre han respetado lo establecido en los acuerdos. Ejemplo de ello es que si bien la reunión de junio de 2016 de la EBITAN (donde se aprobó un nuevo reglamento) fue en Buenos Aires (véase Diario de Cuyo, 2016), la reunión de mayo había tenido lugar en San Juan (véase Tiempo de San Juan, 2016). 


\section{Corolario}

Desde mediados de la década de los ochenta e impulsados por el regreso a la democracia, Argentina y Chile han iniciado un proceso de profundización de la integración en el cual se generaron las condiciones para la institucionalización de la actividad paradiplomática, algo que se vio fuertemente impulsado luego del cambio de milenio. Entre los distintos canales a los que actualmente pueden recurrir los gobiernos no centrales de los dos países, las acciones coordinadas a través de instituciones establecidas por acuerdo bilateral presentan una buena oportunidad para el desarrollo de actividades paradiplomáticas.

A través de la firma de los distintos acuerdos, siendo el Tratado de Paz y Amistad de 1984 la semilla del germen - pero el Tratado de Maipú de 2009 la piedra angular-, los países consolidaron la presencia subestatal en instituciones como los comités de integración -en que se les reconoció la participación directa-y las entidades binacionales, donde se les abrió la posibilidad para que reclamasen un lugar en las mesas de negociaciones.

Sin embargo, el aprovechamiento de estas oportunidades por parte de los gobiernos no centrales - en el caso de las entidades binacionales analizadas - no ha sido homogéneo. En tanto la EBITAN incorporó a la Región de Coquimbo como miembro permanente de la entidad, estableció inicialmente las sedes fuera de las capitales nacionales y protegió al componente subestatal, garantizando que su voz fuese tenida en cuenta; ello no ocurrió en EBIFETRA. El reglamento de la segunda, que fue suscrito el mismo año que el de la primera, establece una estructura organizacional mucho menos compleja. En primer lugar, mantiene a los representantes establecidos en el Protocolo Complementario al Tratado de Maipú, excluyendo así a la Región de Valparaíso o a cualquier otro representante subestatal de Chile. En segundo lugar, el reglamento de la EBIFETRA mantuvo siempre las sedes de la entidad en Buenos Aires y Santiago. Finalmente, en tanto que para sesionar EBITAN requiere por reglamento la participación de todos los miembros, EBIFETRA puede sesionar con solo dos de estos. Es decir, no se buscó incorporar a la región chilena, al tiempo que no se protegió a la provincia argentina de la posibilidad de que los ministerios centrales sesionen en Buenos Aires sin su participación.

Efectivamente, la letra de los acuerdos no es siempre lo que se refleja en la práctica y también son muchos los obstáculos que la región de Coquimbo enfrentó para consolidar su lugar en la mesa de negociación de la EBITAN. No obstante, su motivación logró que el asiento le fuese establecido por reglamento, algo que no se replicó en el caso de Valparaíso. 
Argentina y Chile ofrecieron la misma agencia a proyectos similares; sin embargo, el componente paradiplomático se desarrolló de forma diferenciada en ambos debido al accionar del agente. Se ha normalizado un enfoque en el cual el aumento de la actividad paradiplomática es presentado como una lucha directa contra el gobierno central por mayor participación en los asuntos exteriores. Sin embargo, como se pudo observar en los casos de EBITAN y EBIFETRA, cuando los países ofrecen las posibilidades de expresión paradiplomática, es necesario que los gobiernos no centrales estén dispuestos y motivados para su aprovechamiento, lo que explicaría las diferencias en el desarrollo institucional de las dos entidades binacionales analizadas.

\section{Referencias}

Aguirre, I. (1999). Making sense of paradiplomacy? An intertextual enquiry about a concept in search of a definition. Regional \& Federal Studies, 9(1), 185-209.

Aldecoa, F. y Keating, M. (2000). Paradiplomacia: las relaciones internacionales de las regiones. Madrid, España: Marcial Pons.

Álvarez, M. (2016). Paradiplomacia en las relaciones chileno-argentinas: La integración desde Coquimbo y San Juan (tesis doctoral). Universiteit Leiden, Leiden.

Artaza Rouxel, M. (2010). La paradiplomacia de las regiones chilenas y las provincias argentinas. En L. Maira (ed.), La política internacional subnacional en América Latina (pp. 359-366). Buenos Aires, Argentina: Libros del Zorzal.

Bernal Meza, R. (1990). El papel de las regiones en la formulación de la política exterior y potencial de articulación con regiones de países limítrofes. Antecedentes y perspectivas. Integración Latinoamericana, 15(156), 28-39.

Church, J. M. (2011). Argentine-Chilean relations in the shadow of the debt crisis. Bulletin of Latin American Research, 30(2), 148-162.

Colacrai, M. y Zubelzú, G. (1994). Las provincias y sus relaciones externas. ¿Federalización de la política exterior o protagonismo provincial en las relaciones internacionales? Recuperado de http://www.cerir.com.ar.

Cornago, N. (1999). Diplomacy and paradiplomacy in the redefinition of international security: Dimensions of conflict and co-operation. Regional \& Federal Studies, 9(1), 40-57.

92 Si Somos Americanos. Revista de Estudios Transfronterizos 
Cornago, N. (2010). On the Normalization of Sub-State Diplomacy. En D. Criekemans (ed.), Regional sub-state diplomacy today (pp. 11-36). Leiden, Países Bajos: Martinus Nijhoff Publishers.

Duchacek, I. D. (1984). The international dimension of subnational self-government. Publius, 14(4), 5-31.

Ferrero, M. (2005). La glocalización en acción: regionalismo y paradiplomacia en Argentina y el cono sur latinoamericano. Revista Electrónica de Estudios Internacionales, 11, 1-22.

Gasol Varela, C. (2012). Gobiernos locales y gestión internacional: El caso argentino. Temas de Política Exterior, Comercio y Relaciones Internacionales, 5(5), 133-154.

Hocking, B. (1986). Regional governments and international affairs: Foreign policy problem or deviant behaviour? International Journal, 41(3), 477-506.

Iglesias, E., Zubelzú, G. e Iglesias, V. (eds.). (2008). Las provincias argentinas en el escenario internacional: Desafíos y obstáculos de un sistema federal. Buenos Aires, Argentina: PNUD.

Keating, M. (1999). Regions and international affairs: Motives, opportunities and strategies. Regional \& Federal Studies, 9(1), 1-16.

Keating, M. (2000). Paradiplomacy and Regional Networking. Recuperado de Forum of Federations: http://www.forumfed.org.

Keating, M. (2003). Paradiplomacia y constitución de redes regionales. Revista Valenciana D’Estudis Autonòmics, 36, 39-50.

Kincaid, J. (1990). State and local governments go international. Intergovernmental Perspective, 16(2), 6-10.

Kincaid, J. (2003). Foreign relations of sub-national units. En R. J. Blindenbacher y A. Koller (eds.), Federalism in a changing world: Learning from each other (pp. 74-96). Montreal, Canada: McGill-Queen’s University Press.

Kuznetsov, A. S. (2014). Theory and practice of paradiplomacy. Subnational governments in international affairs. New York, NY: Routledge. 
Maira, L. (ed.). (2010). La política internacional subnacional en América Latina. Buenos Aires, Argentina: Libros del Zorzal.

María Hernández, A. (2003). Rol de las regiones, provincias y municipios en el proceso de integración. En A. M. Hernández y D. Valadés (eds.), Estudios sobre federalismo, justicia, democracia y derechos humanos. Homenaje a Pedro J. Fría (pp. 219-272). México, D.F.: Universidad Nacional Autónoma de México.

Michelmann, H. J. (1990). Conclusion. En H. J. Michelmann y P. Soldatos (eds.), Federalism and international relations: The role of subnational units (pp. 299-315). Oxford, UK: Clarendon Press.

Michelmann, H. J. (2009). Introduction. En H. J. Michelmann (ed.), Foreign relations in federal countries (pp. 3-8). Montreal, Canada: Forum of Federations e International Association of Centers for Federal Studies; Montreal, Canada: McGill-Queen's University Press.

Michelmann, H. J. y Soldatos, P. (eds.). (1990). Federalism and international relations: The role of subnational units. Oxford, UK: Clarendon Press.

Natalizio, M. V. (2007). La multicausalidad y multidimensionalidad de la acción internacional de los actores subnacionales argentinos. El caso de la Ciudad Autónoma de Buenos Aires. Buenos Aires, Argentina: CAEI.

Protocolo complementario al Tratado de Maipú de Integración y Cooperación sobre la constitución de la Entidad Binacional para el proyecto "Túnel Internacional Paso de Agua Negra”, Argentina-Chile, 2010. Recuperado desde http://tratados.cancilleria. gob.ar

Protocolo complementario al Tratado de Maipú de Integración y Cooperación sobre la constitución de la Entidad Binacional para el proyecto "Túnel de Baja Altura Ferrocarril Trasandino Central”, Argentina-Chile, 2010. Recuperado desde http:// tratados.cancilleria.gob.ar

Redacción Diario de Cuyo (9 de junio de 2016). Aprobaron el reglamento interno para el funcionamiento del Túnel de Agua Negra. Diario de Cuyo. Recuperado de: http:// www.diariodecuyo.com.ar.

Schnake, L. (2011). Paradiplomacia en Chile: El caso de la Región Metropolitana. Recuperado de: Bibliothek der Friedrich-Ebert-Stiftung: http://library.fes.de.

94 Si Somos Americanos. Revista de Estudios Transfronterizos 
Silva Soura, V. y Morán León, C. (2010). Relaciones subnacionales. El caso de Chile y Argentina. En L. Maira (ed.), La política internacional subnacional en América Latina (pp. 259-304). Buenos Aires, Argentina: Libros del Zorzal.

Tiempo de San Juan (13 de mayo de 2016). Sesiona EBITAN en San Juan para afinar detalles por el túnel de Agua Negra. Tiempo de San Juan. Recuperado de: http://www. tiempodesanjuan.com.

Tratado de Maipú de Integración y Cooperación, Argentina-Chile, 2009. Recuperado desde http://tratados.cancilleria.gob.ar

Tsebelis, G. (1990). Nested Games. Rational choice in comparative politics. Berkeley, CA: University of California Press.

Tussie, D. (2004). La política comercial en un contexto de federalismo: notas para el estudio del caso argentino. Integración \& Comercio, 21, 69-84.

Zubelzú, G. e Iglesias, V. (2005a). Segundo informe de relevamiento y diagnóstico, parte II. Proyecto: Provincias y Relaciones Internacionales. Recuperado de Consejo Argentino para las Relaciones Internacionales: www.cari.org.ar/pdf/provincias-informe2-parte2. pdf.

Zubelzú, G. e Iglesias, V. (2005b). Segundo informe de relevamiento y diagnóstico, parte I. Proyecto: Provincias y Relaciones Internacionales. Recuperado de Consejo Argentino para las Relaciones Internacionales: http://www.cari.org.ar.

Zurbriggen, C. (2006). El institucionalismo centrado en los actores: Una perspectiva analítica en el estudio de las políticas públicas. Revista de Ciencia Política, 26(1), 67-83. 